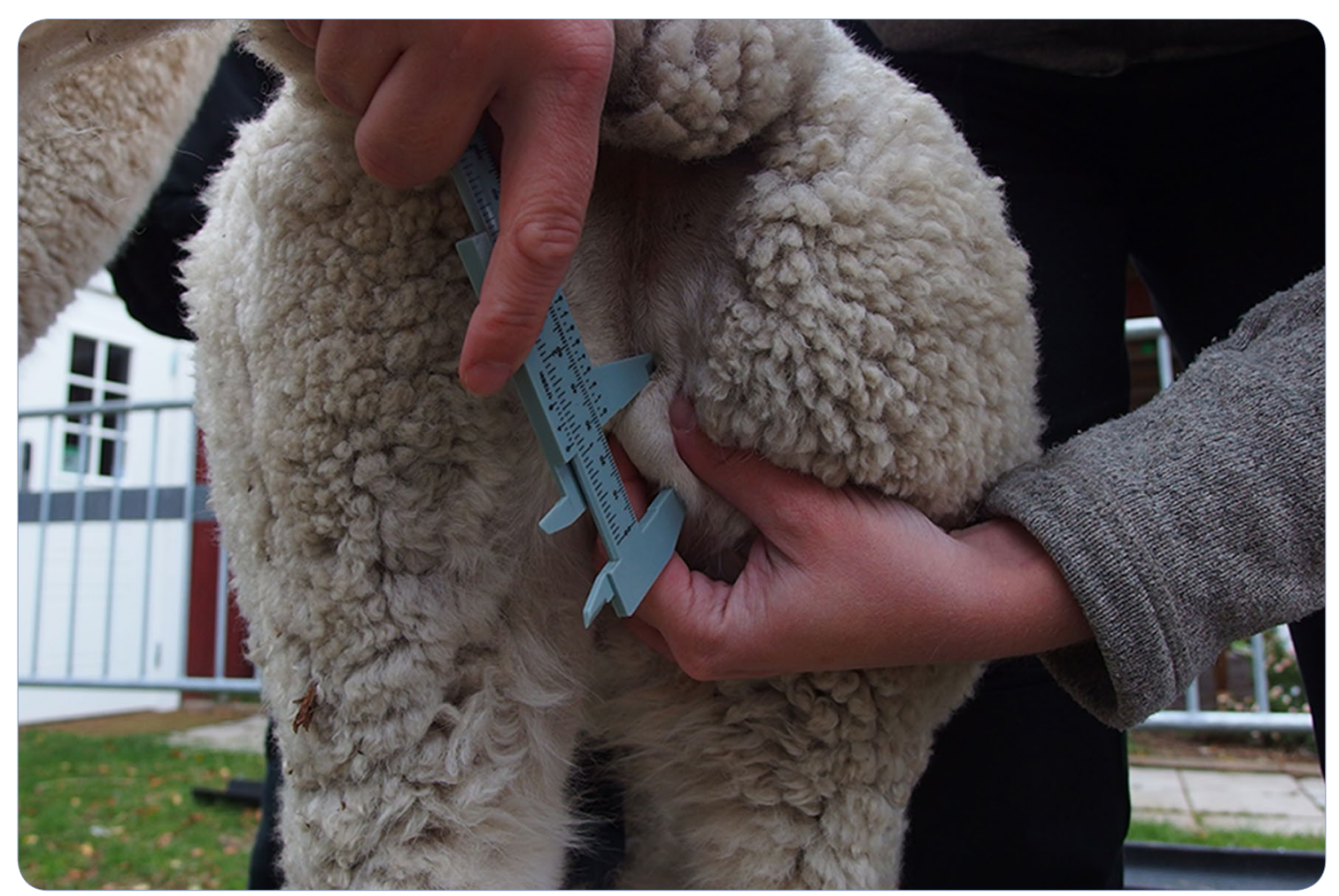

\title{
Testicular length as an indicator of the onset of sperm production in alpacas under Swedish conditions
}

Abraham et al. 


\title{
Testicular length as an indicator of the onset of sperm production in alpacas under Swedish conditions
}

\author{
Maria Celina Abraham', Johanna Puhakka', Alejandro Ruete², Essraa Mohsen Al-Essawe ${ }^{1}$ Kerstin de Verdier ${ }^{3}$, \\ Jane Margaret Morrell ${ }^{1 *}$ and Renée Båge ${ }^{1}$
}

\begin{abstract}
Background: The popularity of alpacas (Vicugna pacos) is increasing in Sweden as well as in other countries; however, knowledge about optimal management practices under Swedish conditions is still limited. The wide age range reported when the onset of puberty can occur, between 1 and 3 years of age, makes management decisions difficult and may be influenced by the conditions under which the alpacas are kept. The aim of this study was to find out when Swedish alpacas can be expected to start producing sperm, by using testicular length and body condition score as a more precise indirect indicator than age.

Results: This study suggests that animals with a testicular length $\geq 3.8 \mathrm{~cm}$ would be producing sperm; however, if it is crucial to know that there is no sperm production for management purposes, the threshold level for testicular length used to differentiate between sperm-producing and non-sperm producing animals should be $\leq 1.6 \mathrm{~cm}$ instead. If only one variable is considered, testicular length appears to better than age alone to predict sperm production. Body condition score together with testicular length explains the individual onset of puberty and better guide management recommendations.
\end{abstract}

Conclusions: Using a combination of these parameters (testicular length, body condition score and age) as a tool for decision making for alpaca husbandry under Swedish conditions is suggested.

Keywords: Vicugna pacos, Puberty, Animal husbandry, Statistical models, Decision making tools

\section{Background}

Alpacas, llamas, guanacos and vicunas are South American camelids and members of the Camelidae family. Alpacas and llamas are domesticated, being kept as production animals (for fibre and meat production, as pack animals, etc.) as well as companion animals. In recent years, the international interest in breeding alpacas has increased together with the demand for more accurate information about their health care and animal husbandry. In Sweden, alpacas have become progressively more popular during the last decade; however, knowledge about optimal management practices and disease

\footnotetext{
*Correspondence: jane.morrell@slu.se

${ }^{1}$ Division of Reproduction, Department of Clinical Sciences, Swedish University of Agricultural Sciences, Box 7054, 75007 Uppsala, Sweden Full list of author information is available at the end of the article
}

panorama under Swedish conditions is still limited [1, 2]. Alpacas present several physiological peculiarities compared with other domestic species. They are not considered to have a breeding season [3]. Like other camelids they are induced ovulators, with continuous waves of follicular growth throughout the year [4]. However the female may reject the male's attempts to mate depending on the stage of follicular growth [3]. There are no reports of males showing seasonality in their willingness to mate.

It is known that nutritional status could influence the onset of puberty in other species [5] but according to Van Saun [6] studies on nutrition in alpacas and its relationship with reproduction are still lacking. Galloway [7] reported that there is a correlation between body size and testicular size, although the wide variation in testicular size suggests that other factors, probably genetic, are 
also important. Previous studies have shown that testicular size could be an indicator of sperm production and hence fertility, which is consistent with observations on the bull, ram and stallion $[7,8]$.

In addition to their physiology, they also have an anatomical difference. The prepuce has adherence to the glans penis until 2 or 3 years of age, making protrusion of the penis impossible in young males $[9,10]$. The adherence disappears gradually when the animal matures, apparently in relation to testosterone levels $[9,11]$.

The detection of sperm production in males is an important factor in managing alpacas for several reasons, among them: (1) owners need to know when young males and females should be separated to avoid undesirable matings, (2) to use good males strategically in breeding programs, (3) to recommend when males should be castrated if they are not going to be used for breeding, (4) for research purposes, to know when it is the best moment to collect samples in order to access useful material, and (5) in a broader perspective, as an indicator of animal welfare.

Research in other countries indicates that male alpacas start producing sperm between 1 and 3 years of age $[7,9]$. This wide range is probably influenced by the conditions under which alpacas are kept, and makes management decisions difficult. It is not known if the information generated in other countries, such as Perú, Australia and USA is applicable to Europe. Therefore, the aim of this study was to investigate with higher precision when Swedish alpacas can be expected to start producing sperm, by using the testicular length as an indirect indicator of the onset of sperm production. Two experiments were performed: Experiment 1 (on farm) to measure testicular size and Experiment 2 to determine the presence of sperm post castration or in cadavers. Whether this age could be affected by the body condition of the animal was also investigated.

\section{Methods}

\section{Experiment 1}

A total of 72 male alpacas, 13-48 months of age (median of 25.5 months), from 11 Swedish farms, were studied during September 2014. Farms were selected for convenience based on geographical distribution and number of animals. The length of the testicles was measured individually with a calliper (Biltema, Sweden) (Fig. 1).

Body condition was scored according to a standardized scale from $1-5$, where 1 is emaciated and 5 is obese [12, 13].

Body condition scoring (BCS) was done by palpating over the central backbone near the last ribs. All of the testicle measurements and BCS were performed by the same operator.

\section{Experiment 2}

\section{Testicles from routine castrations}

Twenty-two pairs of testicles were obtained from onfarm routine castrations of male alpacas on eight Swedish farms. The age of the animals ranged between 11 and 113 months (1-9 years) with a median age of 23 months. The study was conducted from May 2013 to April 2015.

The pairs of testicles were placed in plastic bags with phosphate-buffered saline solution (KV-laboratory, SLU) and were transported to the laboratory in styrofoam boxes containing a cold pack at $4{ }^{\circ} \mathrm{C}$, where they arrived within $24-48 \mathrm{~h}$ of surgery.

\section{Testicles from cadavers}

Six pairs of testicles were obtained at necropsy from male alpacas originating from five Swedish farms. The age of the animals ranged between 16 and 96 months (1-8 years, median age of 42 months). The post mortem evaluations were conducted at Eurofins in Skara (Sweden) during October 2014-February 2015. The pairs of testicles were placed in plastic bags and were transported to the laboratory, where they arrived within a week of death. None of these animals died because of reproductive diseases.

\section{Procedure: measurement of testicles}

After measuring the length of the testicles with a ruler, the tunica albuginea, connective tissues and blood vessels were removed and the epididymides were separated from the testes. The cauda epididymides were isolated, cut into 4-5 pieces and placed in a petri dish, in $1 \mathrm{ml}$ of prewarmed semen extender AndroMed (Minitüb, Tiefenbach, Germany) or INRA 96 (IMV Technologies, L'Aigle, France) for samples from castrations and in $1 \mathrm{ml}$ of PBS for samples from necropsies. After $10 \mathrm{~min}$ of incubation at $37{ }^{\circ} \mathrm{C}$ in $5 \% \mathrm{CO}_{2}$ in an incubator, the presence of spermatozoa was verified using a phase contrast microscope Olympus BX 51 (Olympus, Japan) with 20 and $40 \times$ objectives.

\section{Statistical analysis}

\section{Testicle size}

The relationship between mean testicle length and the individual's age was analysed. We assumed that the mean testicle length follows a normal distribution $\left[L_{i} \sim\right.$ Normal $\left.\left(\mu_{\mathrm{i}}, \sigma\right)\right]$ for each individual $i$ and increases over time given by:

$$
\mu_{i}=\frac{\alpha}{1+e^{-\beta \cdot\left(A g e_{i}-\gamma\right)}}
$$

where $\alpha$ is the maximum mean testicular length, $\beta$ and $\gamma$ are free parameters determining the testicle length increment rate. The parameters where estimated under the 
Bayesian framework in JAGS 3.4 [14], because of its flexibility to estimate parameters uncertainty.

For samples obtained from experiment 1, we also tested the effect of body condition $\left(B C S_{i}\right)$ on the mean testicle length increment rate parameters $(\beta, \gamma)$ as,

$$
\mu_{i}=\frac{\alpha}{1+e^{-\beta \cdot\left(A g e_{i}-\gamma\right) \cdot B C S_{i} \cdot \delta}}
$$

using the mode of the posterior probability distribution of estimates for parameters $\beta$ and letting the algorithms estimate $\alpha, \gamma$ and $\delta$.

The models were evaluated for fit (using the deviance information criteria scores i.e. DIC, Bayesian analogues to the more common Akaike Information Criteria; [15]), and checking the posterior probability distribution.

\section{Sperm presence (logistic models)}

We tested for the effect of age and mean testicle length on the presence of sperm in testicles from necropsies using logistic generalized linear models using the glm (family = "Binomial") function on R v3.2 [16]. Model fit was evaluated through each model's $R^{2}$, comparing the model's fit through AIC [17] and checking the statistical significance of the estimated parameter.

\section{Results}

The distribution of mean testicular length by age groups in Experiment 1 is presented in Table 1. The distribution of mean testicular length by age groups and the presence of sperm in the testicles from castrations and cadavers (Experiment 2) are presented in Table 2.

The testicle length increment model explained the variability in testicular length through time (Table 3 ). Estimates of increment rate parameters $(\beta$ and $\gamma$ ) were similar between the experiments and the difference in maximum mean testicle length $(\alpha)$ is coherent with the expected difference in testicle length due to the collection methodology (Fig. 2). There was, however, large variation in maximum mean testicular length in samples from experiment 1 at greater ages, given the lack of data points in animals older than 50 months. The variability in samples from experiment 2 was generally higher than for experiment 1 but consistent throughout the age range (Fig. 2).

The BCS of the animals ranged between 2.5 and 5 with a median value of 4 . A large variation between different ages was found. The length increment rate of testicles from experiment 1 was positively affected by BCS, while the maximum mean testicle length remained within the same range (Fig. 3).

There is high variability in mean testicle size 10 and 35 months (Figs. 2, 3). Indeed, the probability of sperm presence is better explained by mean testicular length

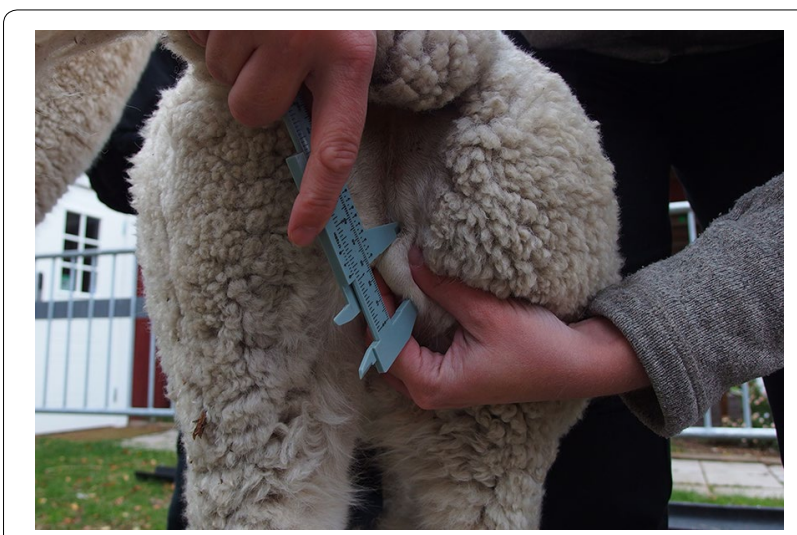

Fig. 1 Measurement of testicles with a calliper

$\left(\mathrm{p}=0.01 ; \mathrm{R}^{2}=0.77 ; \mathrm{AIC}=14.97 ;\right.$ Fig. $\left.4 \mathrm{a}\right)$ than by the animals' age $\left(p=0.047 ; R^{2}=0.38\right.$, AIC $=27.87$; Fig. $\left.4 b\right)$.

\section{Discussion}

The wide range when the onset of puberty can occur, between 1 and 3 years of age, makes management decisions difficult and may be influenced by the conditions under which the alpacas are kept. The aim of this study was to find out if the same range could be expected in Europe (particularly in Sweden) as in other parts of the world. By using a model of testicular length that accounts for variability in age and BCS (Figs. 2 and 3), we tried to predict when alpacas can be expected to start producing sperm. As expected, we found that if only one parameter is considered, testicular length is better than age alone to estimate sperm production (Fig. 4) but that the inclusion of BCS and age together with testicular length gives better predictability than a single parameter. Therefore, we suggest using a combination of these parameters as a tool for decision making in the selection of potential male sires.

Our results showed a large variation in mean testicular length and the presence of sperm at different ages. These findings are consistent with Galloway [7]. However, the number of observations in the upper end of the range is small; it would be beneficial for our model to include data from more animals at a greater age or with larger testicles (Fig. 2) but such animals were scarce in our study because of the owners' decision when to castrate their alpacas. From our observations (Fig. 4) we would expect that animals with a testicular length $\geq 3.8 \mathrm{~cm}$ would be producing sperm (testicular length at which the median probability of sperm presence is $\geq 0.99$ ); however, if it is crucial to know that there is no sperm production for management purposes, the threshold level for testicular length used to differentiate between sperm-producing and non-sperm producing animals should be $\leq 1.6 \mathrm{~cm}$ 
instead (testicular length at which the median probability of sperm presence is $\leq 0.01$ ).

\section{Measurement of the testicles}

Due to the small size of the alpaca testicles, their location very close to the abdomen and the difficulties in restraining the animals, only testicular length was measured in live animals in this study. According to Galloway [7] testicular length is a good measurement of testicle size in alpacas and it is easy to assess accurately. In bulls, measurement of the scrotal circumference is used for evaluating sperm-producing ability, because this is an objective measure, regardless of the operator, and has a strong correlation to the testicle size [18]. However, scrotal circumference is difficult to measure in alpacas due to their anatomical characteristics.

In our study, all the testicle measurements and BCS assessments were done by the same person, to minimize possible methodological errors. It should be noted that the testicular length taken from castrations or cadavers did not include the scrotal layers whereas, of necessity, the measurements on living animals did include the scrotum. Despite this difference in methodology, the measurements followed the same pattern.

\section{Puberty}

Puberty is not a single point in time, but an ongoing process requiring endocrinological and behavioural components as well as anatomical and neurological development. In the literature there are different definitions of puberty, including the presence of fully developed genitalia, libido and a minimum concentration of sperm in the ejaculate. Galloway (personal communication, 2014) defines puberty in male alpacas as the moment when, for the first time, a male can mate a female and produce a pregnancy. For this to be possible, sperm production and good libido are necessary, and the penis should be completely free of adhesions. Although young males often begin to show interest in females and can display mounting behaviour around the age of one year, most of them are not able to carry out a mating at this age $[19,20]$. Complete erection and intromission is only possible when the preputial attachments to the penis have totally disappeared [10]. The detachment seems to be affected positively by the level of testosterone [21]. Early detachment is a desirable genetic trait in young animals and therefore should be an important parameter in the evaluation of the breeding potential of males, together with large testis size $[7,10,22]$ which is assumed to be directly related to sperm production, as in other ruminants [20]. In addition, it is important to know what testicular size should be considered as normal
Table 1 Mean testicular length and number of alpacas by age group (Experiment 1, in vivo measurements)

\begin{tabular}{lcccc}
\hline $\begin{array}{l}\text { Mean length } \\
(\mathbf{c m})\end{array}$ & $\mathbf{1 2 - 2 3}$ months & $\mathbf{2 4 - 3 5}$ months & $\mathbf{3 6 - 4 8}$ months & Total \\
\hline$<3$ & 8 & 0 & 0 & 8 \\
$3-3.9$ & 14 & 8 & 0 & 22 \\
$>4$ & 8 & 24 & 10 & 42 \\
Total & 30 & 32 & 10 & 72 \\
\hline
\end{tabular}

Table 2 Mean testicular length, number of alpacas by age group and sperm presence (Experiment 2, testicles from castration $n=22$; testicles from cadavers $n=6$ )

\begin{tabular}{llll}
\hline Mean length $(\mathbf{c m})$ & Age $^{\mathbf{a}}$ (months) & \multicolumn{2}{l}{ Sperm presence (\%) } \\
\cline { 3 - 4 } & & Castration & Cadavers \\
\hline$<3$ & $12-23$ & $1 / 6(17)$ & $0 / 3(0)$ \\
& $24-35$ & $1 / 4(25)$ & - \\
$3-3.9$ & $>36$ & $1 / 1(100)$ & - \\
& $12-23$ & $3 / 4(75)$ & - \\
& $24-35$ & $3 / 3(100)$ & - \\
$>4$ & $>36$ & - & - \\
& $12-23$ & - & - \\
& $24-35$ & - & - \\
& $>36$ & $4 / 4(100)$ & $3 / 3(100)$ \\
\hline
\end{tabular}

${ }^{a}$ Age at castration/necropsy

for the identification of pathological changes, e.g., hypoplasia or degeneration.

According to Tibary [23] the age at puberty varies depending on, among other factors, genetics, nutritional status, climate, and at what time of year the individual was born. Testicular volume may change according to environmental temperature [24]. Therefore, it would be interesting to do additional measurements of testicular length and body condition score in Sweden at different seasons of the year. Although studies suggest that female alpacas may show some seasonality in their willingness to mate, at least in the Peruvian Andes [25], there have not been any reports on the effect of season on male libido, as an indirect indicator of testosterone production. Moreover, if further studies on testicular measurements are to be made, it would be desirable to include a check for the breakdown of the preputial attachments, to provide additional information on the likelihood that mating can take place.

\section{Nutritional status/BCS}

Peruvian alpacas have smaller testicles than Australian alpacas probably due to a poorer nutritional status [7]. Our results are in line with Galloway's report, 
Table 3 Model fit (DIC and Delta DIC) and parameter estimates for testicle length of Swedish alpacas

\begin{tabular}{lcclll}
\hline Model & DIC & Delta DIC & $\mathbf{a}$ & $\boldsymbol{\beta}$ & $\boldsymbol{\gamma}$ \\
\hline Experiment 1 & & & & & \\
$\quad$ Null & 160.8 & 0 & $3.97(3.8-4.14)$ & & \\
Increment & 117.3 & 43.5 & $4.96(4.33-8.445)$ & $0.078(0.019-0.26)$ & $5.89(-0.87-29.77)$ \\
Increment + BCS & 116.7 & 0.6 & $4.75(4.32-6.45)$ & 0.078 & $5.4(0.56-10.25)$ \\
Experiment 2 & & & & & $0.35(0.10-1.02)$ \\
$\quad$ Null & 87.8 & 0 & $2.97(2.55-3.39)$ & & $15.47(5.17-31.50)$ \\
Increment & 66.9 & 20.9 & $4.43(3.67-5.95)$ & $0.069(0.02-0.16)$ & \\
\hline
\end{tabular}

Values are (median $95 \% \mathrm{Cl}$ in brackets). DIC scores are compared to the null model (including only an intercept parameter). For data from experiment 1, the model including body condition score (BCS) is compared to the increment model. Experiment $1, n=72$. Experiment $2, n=28$

$a$ is the maximum mean testicular length

$\beta$ and $\gamma$ are free parameters determining the testicle length increment rate

$\delta B C S$ effect on increment rate

probably because alpaca husbandry in Sweden is likely to be more similar to Australian conditions than Peruvian conditions, especially in terms of nutrition and thus body weight. In this study, a significant positive effect of body condition on the testicle length increment rate was observed.

Fowler [26] indicates that overweight is probably a more common problem than emaciation in alpacas in the Western world, although a study carried out in Sweden by Björklund [2] found that emaciation can be a problem in weanling alpacas. It is worth mentioning that there are two different systems of production of South American camelids in the world: the traditional Andean herding strategies, in a pastoral economy in the dry highlands, with a high altitude grassland between 3000 and $4800 \mathrm{~m}$ above sea level, and the other system under very different and more favourable conditions, at a low altitude of no more than $800 \mathrm{~m}$ above sea level [27]. These husbandry conditions are likely to result in differing availability of nutrients and thus possibly the onset of puberty.

It is known that decreased nutrient intake delays the onset of puberty in bulls and that, conversely, a diet with high energy level produce a faster development of the testicles $[28,29]$. However, there is little information regarding the effects of diet on reproductive function in male alpacas $[6,30]$. It has been shown that certain parameters, including semen volume and sperm concentration

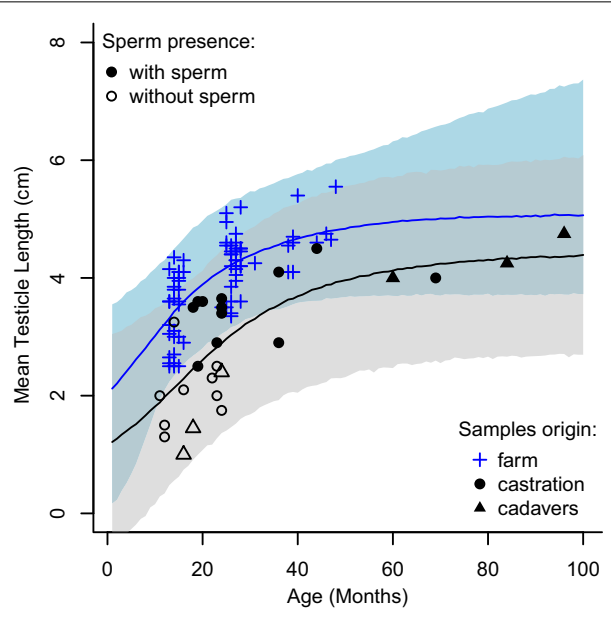

Fig. 2 Length increment model for alpaca testicle length. Experiment 1, "farm", $n=72$; solid line (mean), and dark grey polygon (95\% $\mathrm{Cl}$ ); Experiment 2 (from castrations $(n=22)$ and cadavers $(n=6)$; dashed line (mean) and light grey polygon ( $95 \% \mathrm{Cl}$ ). Note: the measurements on living animals included the scrotal layers whereas organs obtained after castration or from cadavers did not

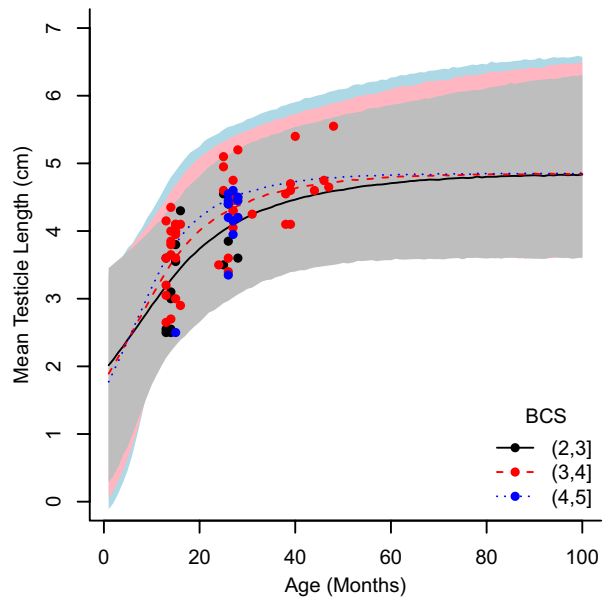

Fig. 3 Length increment model for alpaca testicle length, incorporating age and body condition score (BCS). Testicle length measured in vivo (Experiment $1 ; n=72$ ); Circles represent individual values, lines represent means. Black line and circle = body condition score range from 2 to 3 ; red line and circle = body condition score range from 3 to 4 , blue line and circle = body condition score range from 4 to 5 . Shaded area indicates $95 \%$ confidence interval 

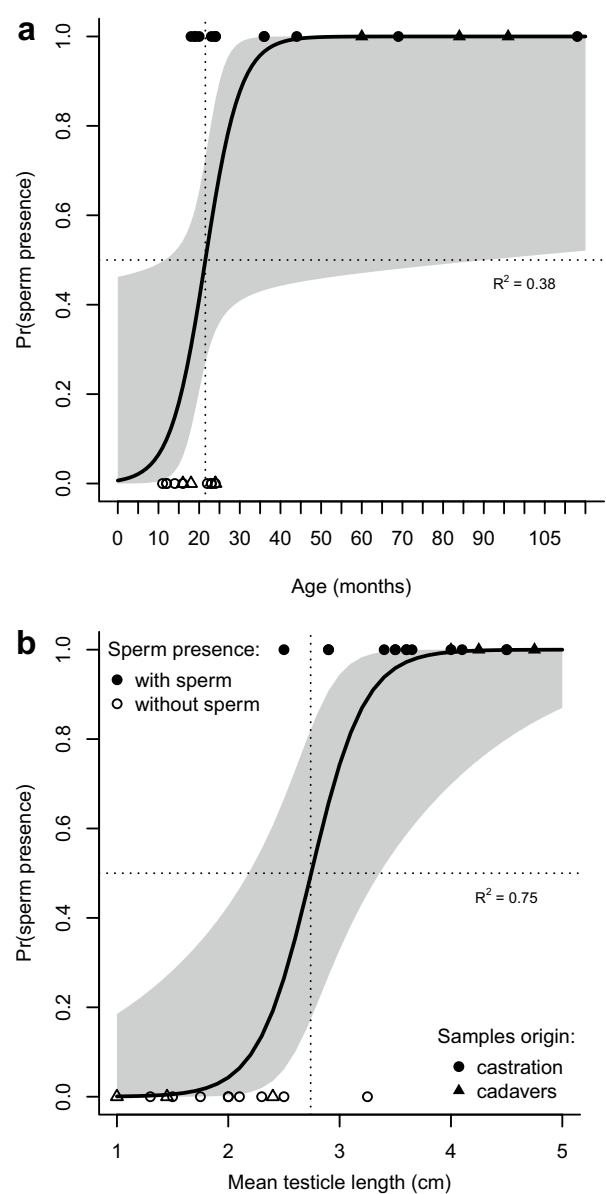

Fig. 4 Probability of sperm presence in alpaca testicles. Samples from Experiment $2(n=28$ ) explained by $\mathbf{a}$ age, and $\mathbf{b}$ mean testicle length. Black circles represent organs from castrations, black triangles represent organs from cadavers; filled circles represent organs containing sperm, open circles represent organs without sperm. Animals with testicular length $\geq 3.8 \mathrm{~cm}$ would be producing sperm (median probability of sperm presence is $\geq 0.99$ ); and animals with testicular length $\leq 1.6 \mathrm{~cm}$ will not be producing sperm (median probability of sperm presence is $\geq 0.01$ )

as well as the biochemical composition of semen may vary depending on the feeding, but it is unclear if this has significance in practice [30].

\section{Breeding management}

By detecting puberty in males as early as possible, owners are able to separate females and males to avoid undesirable matings, to use elite males strategically in breeding programs, and to castrate the ones that are not intended to be used as stud males.

When selecting males for breeding, owners should select early maturing males with large testicular size [7, 31]. Selecting for large testicular size, together with an evaluation of the sexual behavior, BCS and the detachment of preputial adherences, is likely to improve male reproductive efficiency.

Our study supports the recommendations of Vaughan [31]: Breeders/owners are encouraged to measure testicular length and BCS every 6 months, from 12 to 36 months of age, to assist with selection of potential male sires.

\section{Conclusions}

If only one parameter is considered, testicular length is better than age alone to predict sperm production. However, the inclusion of BCS and age gives better predictability than a single parameter.

Our results suggests that animals with a testicular length $\geq 3.8 \mathrm{~cm}$ would be producing sperm; however, if it is crucial to know that there is no sperm production for management purposes, the threshold level for testicular length used to differentiate between sperm-producing and non-sperm producing animals should be $\leq 1.6 \mathrm{~cm}$ instead.

A combination of these parameters as a tool for decision making in the selection of potential male sires for animal husbandry under Swedish conditions is suggested.

\section{Authors' contributions}

$J P, M C A, E M A$ and JMM were involved in data collection; MCA, JP, KV, JMM, RB participated in the design and coordination of the study and helped to draft the manuscript. AR participated in the design of the study and performed the statistical analysis. All authors read and approved the final manuscript.

\section{Author details}

${ }^{1}$ Division of Reproduction, Department of Clinical Sciences, Swedish University of Agricultural Sciences, Box 7054, 75007 Uppsala, Sweden. ${ }^{2}$ Department of Ecology, Swedish University of Agricultural Sciences, Box 7044, 75007 Uppsala, Sweden. ${ }^{3}$ National Veterinary Institute, 75189 Uppsala, Sweden.

\section{Acknowledgements}

MCA is funded by a grant awarded to Professor Jane Morrell by the Veterinary Faculty Swedish University of Agricultural Sciences; Alpaca owners and veterinarians for sending the samples and allowing us to work with their animals; Panisara Kunkitti for help in the laboratory; SVA and Eurofins for the samples from necropsies; Professor emeritus Lennart Söderquist for valuable discussions and critically reading the manuscript.

\section{Competing interests}

The authors declare that they have no competing interests.

\section{Ethics approval}

This study did not require official or institutional ethical approval. The animals were handled according to good ethical standards and Swedish legislation.

Received: 25 November 2015 Accepted: 17 January 2016

Published online: 02 February 2016

\section{References}

1. de Verdier K, Bornstein S. Alpackor i Sverige —en ny utmaning (in Swedish). (Alpacas in Sweden- a new challenge). Svensk Veterinärtidning. Swed Vet J. 2010;1:19-23. 
2. Björklund C. Diseases and causes of death among camelids in Sweden: a retrospective study of necropsy cases 2001-2013. Department of Clinical Sciences, Swedish University of Agricultural Sciences Degree project in Veterinary Medicine. 2014.

3. Vaughan J. Ovarian function in South American camelids (alpacas, Ilamas, vicunas, guanacos). Anim Reprod Sci. 2011;124:237-43.

4. Vaughan JL, Macmillan KL. D'Occhio MJ. Ovarian follicular wave characteristics in alpacas. Anim Reprod Sci. 2004;80:353-61.

5. Fernandez-Baca S. La alpaca: Reproducción y crianza (in Spanish). (Reproduction and breeding). Centro de Investigación Instituto Veterinario de Investigaciones Tropicales y de Altura (IVITA). Veterinary Research Center for research in tropical and high-altitude areas). Lima, Peru; 1971. 23-27.

6. Van Saun RJ. Effect of nutrition on reproduction in llamas and alpacas. Theriogenology. 2008;70:508-14.

7. Galloway DB. The Development of the testicles in alpacas in Australia. Australian Alpaca Association. Canberra; 2000. p. 21-3.

8. Thompson DL, Pickett BW, Squires EL, Amann RP. Testicular measurements and reproductive characteristics in stallions. J Reprod Fertil. 1979;27:13-7

9. Sumar J. Studies on reproductive pathology in alpacas. Doctoral Thesis, Department of Clinical Sciences, Swedish University of Agricultural Sciences. 1983.

10. Tibary A, Pearson LK, Anouassi A. Applied andrology in camelids. In: Chenoweth PJ, Lorton SP, editors. Animal andrology: theories and applications. Wallingford: CAB International; 2014. p. 418-49.

11. Bravo PW, Johnson LW. Reproductive physiology of the male camelid. In: Chenoweth PJ, Lorton SP, editors. Applied andrology in camelids animal andrology: theories and applications. Wallingford: CAB International; 2014. p. 418-49.

12. Australian Alpaca Association. Body condition score (BCS) of Alpacas, Alpaca Fact Sheet\#4. 2008. https://www.alpaca.asn.au/index.php/ resources/alpaca-fact-sheets Accessed June 2015.

13. Swedish Animal Health Service (Svenska Djurhälsovården). Hullbedömning av alpackor (in Swedish). (Alpaca body condition scoring) 2010. http://www.svdhv.org/upload/documents/Artiklar/101207_kamel_hullbedomning.pdf. Accessed June 2015.

14. Plummer M. JAGS: A program for analysis of Bayesian graphical models using Gibbs sampling. 2012. http://mcmc-jags.sourceforge.net/ Accessed June 2015.

15. Spiegelhalter DJ, Best NG, Carlin BP, van der Linde A. Bayesian measures of model complexity and fit. J R Stat Soc Series B. 2002;64:583-639.

16. R Development Core Team. R: a language and environment for statistical computing. R Foundation for Statistical Computing, Vienna, Austria. 2014.
17. Burnham KP, Anderson DR. Model selection and multimodel inference. Forts Collins: Springer; 2002.

18. Hahn J, Foote RH, Seidel GE Jr. Testicular growth and related sperm output in dairy bulls. J Anim Sci. 1969;29:41-7.

19. Fernandez-Baca S. Manipulation of reproductive functions in male and female New World camelids. Anim Reprod Sci. 1993;33:307-23.

20. Brown BW. A review on reproduction in South American Camelids. Anim Reprod Sci. 2000;58:169-95.

21. San Miguel C, Leyva V, Garcia VW. Administración de testosterona en alpacas con adherencias pene-prepuciales (in Spanish). (Administration of testosterone in alpacas with penile-preputial adhesions). Rev Inv Vet Peru. 2002;13:28-36.

22. Sumar J. Reproduction in llamas and alpacas. Anim Reprod Sci. 1996;42:405-15

23. Tibary A, Vaughan J. Reproductive physiology and infertility in male South American camelids: a review and clinical observations. Small Ruminant Res. 2006;61:283-98.

24. Stelleta C, Vencato J, Oztutar F, Milani C, Daskin A, Romagnoli S. Seasonal variation of testicular functionality in alpaca (Vicugna pacos) raised in Italy (abstract). Proceedings of the 31st Meeting of the European Embryo Transfer Association (AETE); Belgium, 2015. A029E. http://www.cbra.org. br/portal/publicacoes/ar/2015/arjs2015.htm

25. Winblad von Walter, Amanda, 2015. Inter-species embryo transfer in South American camelids. Epsilon, SLU, Uppsala. http://stud.epsilon.slu. se/7975/.

26. Fowler ME. Medicine and Surgery of Camelids. 3rd ed. Ames: Wiley-Blackwell; 2010.

27. Miragaya MH, Chaves MG, Agüero A. Reproductive biotechnology in South American camelids. Small Ruminant Res. 2006;61:299-310.

28. Nolan CJ, Neuendorff DA, Godfrey RW, Harms PG, Welsh TH, McArthur $\mathrm{NH}$, Dandel RD. Influence of energy intake on prepubertal development of Brahman bulls. J Anim Sci. 1990;68:1087-96.

29. Brito LFC, Barth AD, Wilde RE, Kastelic JP. Effect of growth rate from 6 to 16 months of age on sexual development and reproductive function in beef bulls. Theriogenology. 2012;77:1398-405.

30. Juyena NS, Vencato J, Pasini G, Vazzana I, Stelleta C. Alpaca semen quality in relation to different diets. Reprod Fertil Dev. 2013;25:683-90.

31. Vaughan J. Selection of fertile alpacas, Size really does matter! Alpaca World Magazine. 2005. Available on: http://www.alpacaseller.com/DisplayArticleList.php?PHPSESSID=1r1n6h7k0gbgbk106i8ikk3dt5. Accessed June 2015.

\section{Submit your next manuscript to BioMed Central and we will help you at every step:}

- We accept pre-submission inquiries

- Our selector tool helps you to find the most relevant journal

- We provide round the clock customer support

- Convenient online submission

- Thorough peer review

- Inclusion in PubMed and all major indexing services

- Maximum visibility for your research

Submit your manuscript at www.biomedcentral.com/submit
() Biomed Central 